\title{
Comunicación
}

\section{Estabilización quirúrgica utilizando la fijación segmentaria con clavo de Steinmann y alambre quirúrgico en un canino con luxofractura vertebral toracolumbar}

\author{
Surgical stabilization using segmental fixation with Steinmann nail and surgical \\ wire in a canine with vertebral thoracolumbar luxofracture
}

Ysaac Chipayo G. ${ }^{1}$, Patricia Lozada P. ${ }^{1}$, Juan Olazabal L. ${ }^{2}$, Diego Díaz C. ${ }^{1}$

\section{Resumen}

Se describe el caso clínico de un paciente canino atendido 30 minutos después de ocurrido un accidente automovilístico, con una luxofractura de la vértebra torácica 12 y el tratamiento quirúrgico de la misma a través de una técnica de fijación segmentaria de las vértebras torácicas 11, 12, 13 y lumbar 1, usando un clavo de Steinmann y alambres quirúrgicos. El paciente se presentó con paraplejia y escasa respuesta al estímulo de dolor profundo, la cual, en menos de 24 horas de la primera evaluación se mostró nula. Esto demostraba el rápido deterioro de las funciones medulares detectables en el examen clínico; sin embargo, el manejo rápido y la obtención de imágenes diagnósticas permitió el desarrollo de la técnica quirúrgica antes de las 24 horas de la lesión. El paciente recuperó la postura y la marcha a los tres días posquirúrgicos, logrando deambular a los siete días de la intervención. Estos resultados confirman la necesidad de la oportuna evaluación neurológica con criterio diagnóstico a fin de tomar la decisión quirúrgica adecuada de manera rápida.

Palabras clave: trauma espinal; luxofractura vertebral; fijación segmentaria; canino

\footnotetext{
${ }^{1}$ Clínica de Animales Menores (CAM), Facultad de Medicina Veterinaria, Universidad Nacional Mayor de San Marcos, Lima, Perú

${ }^{2}$ Laboratorio de Bioquímica, Nutrición Animal y Alimentación Animal, Facultad de Medicina Veterinaria, Universidad Nacional Mayor de San Marcos, Lima, Perú

${ }^{3}$ E-mail: ichipayo@hotmail.com
} 
It is described the clinical case of a canine patient attended 30 minutes after a car accident, with a luxofracture of the thoracic vertebra 12 and its surgical treatment by a technique of segmental fixation of the thoracic vertebrae 11,12 13 and lumbar 1, using a Steinmann nail and surgical wires. The patient presented with paraplegia and little response to the deep pain stimulus, which, in less than 24 hours after the first evaluation was null. This demonstrated the rapid deterioration of the medullary functions detectable in the clinical examination; however, the rapid action and the diagnostic images allowed the execution of the surgical technique before 24 hours after the injury. The patient recovered his posture and the march three days after surgery, managing to walk seven days after the intervention. These results confirm the need for a timely neurological evaluation with diagnostic criteria in order to make the appropriate surgical decision quickly.

Key words: spinal trauma; vertebral luxofracture; segmental fixation; canine

\section{INTRODUCCIÓN}

El traumatismo medular agudo causado por fracturas o luxo-fracturas vertebrales representa una parte importante de la casuística neurológica en animales de compañía (Bulcão et al., 2012), y ha sido frecuentemente asignada en canes como la segunda causa de afección medular después de las hernias discales (Shores, 1992; Bagley, 2000).

Las fracturas y luxaciones traumáticas vertebrales caninas generalmente ocurren por accidentes de tránsito y caídas de gran altura (Slatter, 2006). Son inducidas por fuerzas que causan hiperextensión intensa, hiperflexión, compresión axial o rotación vertebral, y se producen a menudo en la unión (o cerca) de un segmento vertebral móvil y uno más rígido (por ejemplo, en la región toracolumbar) (Fossum et al., 2007). La incapacidad de las estructuras de soporte de la columnapara resistir estas fuerzas causa una discontinuidad mecánica, donde el traumatismo puede afectar a una o varias estructuras anatómicas: vértebras, discos intervertebrales, meninges y médula espinal (Jeffery, 2010).
El tratamiento de fracturas inestables implica el uso de técnicas quirúrgicas cuyo objetivo es el realineamiento de las estructuras alteradas, la estabilización rígida, la prevención del trauma repetido al sistema nervioso, la descompresión medular y la óptima reparación de la fractura sin que se forme seudoartrosis (Slatter, 2006). Diversas técnicas de fijación segmentaria para la estabilización de luxofracturas espinales y como tratamiento de columnas escolióticas han sido descritas. Algunas de ellas incluyen el uso de clavos de Steinmann y alambres quirúrgicos (Moshirfar et al., 2005). Harrington (1962) utilizó dos barras paralelas a nivel de los procesos espinosos y ganchos sublaminares como material de sujeción, mientras que Gage (1971) usó un clavo de acero quirúrgico como implante semirígido asegurado por alambres a la espina dorsal. Esta técnica aplicada en pacientes veterinarios ofrece una razonable inmovilización en canes pequeños y en gatos (Kirby, 1995; Voss y Montavon, 2004).

Algunas de las limitaciones más observadas en cuanto al desarrollo y éxito de los tratamientos de luxofracturas vertebrales son el momento del diagnóstico clínico, el peso y 
tamaño del paciente, el tiempo entre la ocurrencia de la lesión y el momento de la intervención y, por otro lado, la renuencia de algunos propietarios a asumir riesgos ante el pronóstico reservado a malo de ciertos pacientes (Pellegrino, 2010). En el presente caso clínico se contempla la posibilidad de resolución de una lesión vertebral inestable, de ocurrencia temprana, en un paciente de bajo peso, usando la fijación segmentaria con materiales que son de fácil disponibilidad y bajo costo, como son los alambres quirúrgicos y el clavo de Steinmann.

\section{Caso Clínico}

Se presentó a consulta en el servicio de emergencia de la Clínica de Animales Menores de la Facultad de Medicina Veterinaria de la Universidad Nacional Mayor de San Marcos, un paciente canino, de raza Perro sin Pelo del Perú, de 2 años y $12 \mathrm{~kg}$ de peso, con historia de haber sido atropellado hace 30 minutos.

\section{Examen Clínico}

En la exploración física se observó estado de alerta, incapacidad de movimiento voluntario del tren posterior, miembros fríos al tacto, mucosas rosado-pálidas, frecuencia cardiaca de $160 \mathrm{lpm}$, ritmo cardiaco regular, frecuencia respiratoria de $50 \mathrm{rpm}$, tiempo de llenado capilar de 1.5-2.0 segundos y pulso débil. El abdomen se mostraba moderadamente tenso a la palpación, presentaba temperatura de $37^{\circ} \mathrm{C}$, y deformidad dorsal de columna toracolumbar (xifosis) con dolor a la palpación.

Los signos neurológicos observados fueron: miembros torácicos sin alteración, ausencia de movimiento voluntario de miembros posteriores, vejiga de fácil vaciado, reflejos patelar, flex or y perineal presentes e incrementados, ausencia de sensación de dolor superficial y escasa respuesta de sen- sación de dolor profundo en miembros posteriores. Estos resultados permitieron el diagnóstico clínico de una paraplejia con lesión de neurona motora superior para los miembros posteriores, situando de manera preliminar la lesión neurológica entre los segmentos medulares T3-L3. Clínicamente se consideró como más probable el compromiso de la región toracolumbar (unión de región móvil con región más estable). Cabe resaltar que al día siguiente de la primera evaluación se observó ausencia de respuesta al estímulo doloroso, lo cual denotaba empeoramiento de la función medular.

El paciente fue inicialmente estabilizado mediante la administración de fluidos cristaloides y Tramadol a dosis de $4 \mathrm{mg} / \mathrm{kg}$ de peso, vía endovenosa. Posteriormente se decidió a utilizar succinato sódico de metilprednisolona a dosis de $30 \mathrm{mg} / \mathrm{kg}$ vía endovenosa. Previamente se suministró omeprazol a dosis de $1 \mathrm{mg} / \mathrm{kg}$ vía endovenosa.

\section{Exámenes Auxiliares}

De acuerdo con los resultados del examen clínico se procedió a evaluar radiográficamente el segmento T3-L3. Se indicó, además, hemograma y bioquímica sanguínea básicos, así como la tomografía computarizada (TC) de columna. La evaluación radiográfica se realizó a través de la incidencia laterolateral de la columna, encontrándose un severo cambio en el eje longitudinal de la columna vertebral en relación a las vértebras T12-T13, generando una imagen radiográfica que se suele diagnosticar como «choque de trenes» (Figura 1). En este caso, la imagen fue producida por la fractura del cuerpo vertebral T12 y la luxación asociada de las articulaciones intervertebrales T12-T13. Las imágenes tomográficas se realizaron en un centro de diagnóstico particular y mostraron la fractura múltiple del cuerpo vertebral T12 y del proceso articular derecho, además de la severa inestabilidad de la articulación T12T13 (Figuras 2A y 2B). 


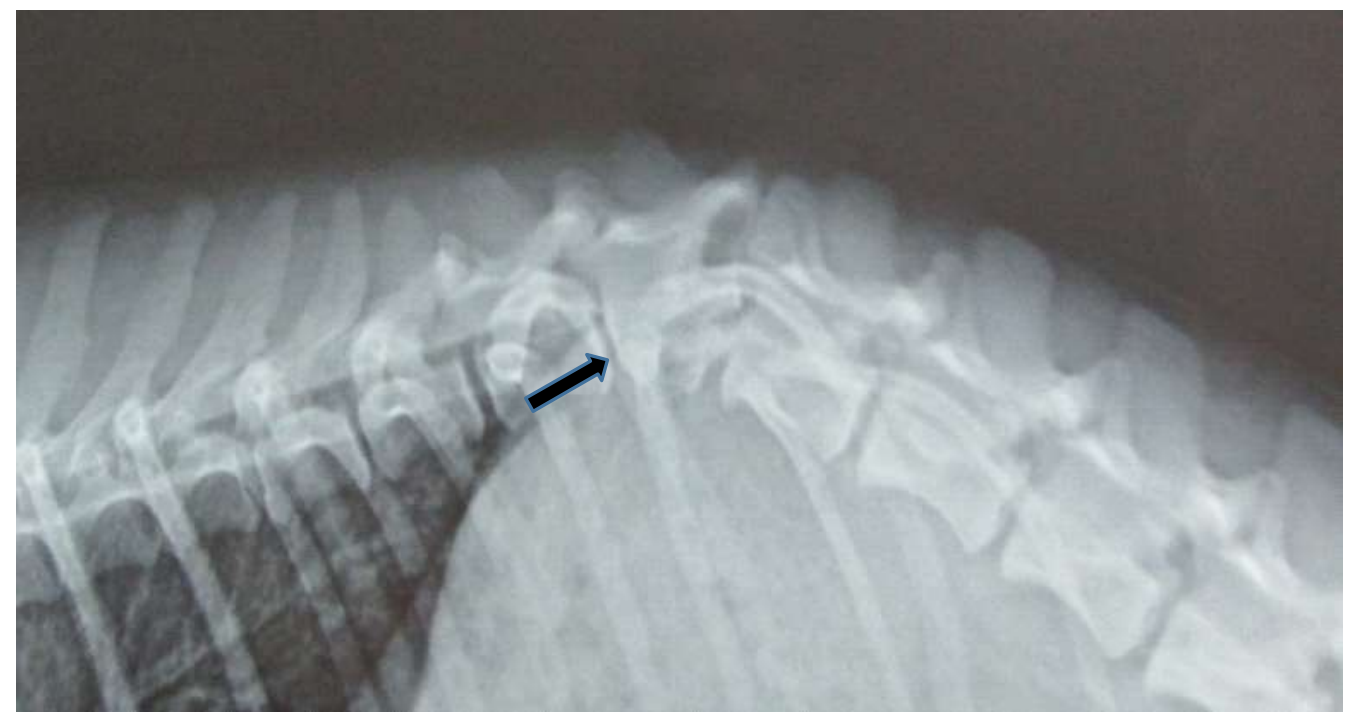

Figura 1. Imagen radiográfica (vista laterolateral de la columna) de un Perro sin Pelo del Perú, de 2 años, con historia reciente de atropello vehicular. La flecha muestra la fractura del cuerpo vertebral T12 y la luxación asociada de las articulaciones intervertebrales T12-T13

Dada la escasez de medios económicos del propietario se decidió implementar una técnica quirúrgica de fijación segmentaria que incluyera el uso de materiales de fácil disponibilidad y bajo costo, como el alambre quirúrgico y clavo de Steinmann, descrita por Gage (1971).

\section{Tratamiento Quirúrgico y Posquirúrgico}

La premedicación anestésica fue realizada por vía endovenosa con midazolam a dosis de $0.5 \mathrm{mg} / \mathrm{kg}$ vía endovenosa, cefalotina, a dosis de $25 \mathrm{mg} / \mathrm{kg}$ vía endovenosa, y fentanilo en bolo de $5 \mu \mathrm{g} / \mathrm{kg}$. Seguidamente se preparó una solución para mantener la medicación analgésica a ritmo de infusión continua a dosis de $5 \mu \mathrm{g} / \mathrm{kg} / \mathrm{h}$ vía endovenosa. La inducción anestésica se realizó con ketamina a dosis de $10 \mathrm{mg} / \mathrm{kg}$ vía endovenosa y para el mantenimiento anestésico se usó anestesia inhalatoria con sevofluorano (Chipayo et al., 2018).
El abordaje de la columna vertebral se realizó por vía dorsal, con una incisión en piel sobre los procesos espinosos desde la vértebra T10-L2. Se continuó con la incisión de la fascia toracolumbar, la separación de los músculos epiaxiales (multífido, torácico y lumbar largo, espinal y semiespinal) y la esqueletización de la porción dorsal de las vértebras T11, T12, T13 y L1. Se realizó la exploración del canal medular ejecutando una variante de la técnica de laminectomía de Funkquist, donde se hace una apertura mínima necesaria de los arcos vertebrales T12T13 para la visualización de la luxofractura y el cordón medular. Con la ayuda de un separador de Gelpi colocado entre los procesos espinosos de las vértebras T11-L1 y con la ayuda de pinzas de campo sosteniendo los mencionados procesos se distrajo de manera delicada las estructuras vertebrales para lograr la alineación del canal medular. Una vez lograda la recuperación del alineamiento longitudinal de las estructuras vertebrales y 

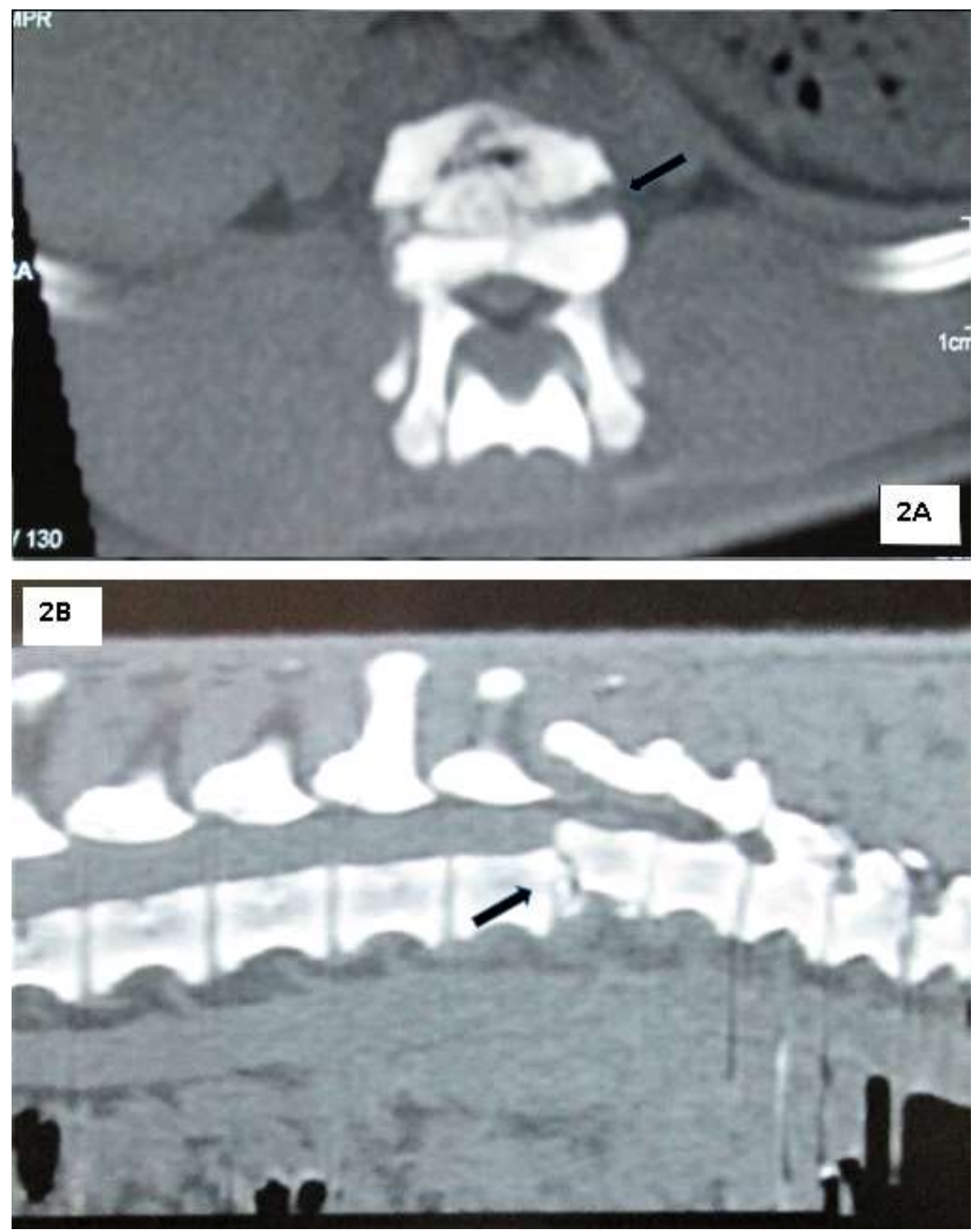

Figura 2. Imágenes tomográficas de paciente canino de 2 años y $12 \mathrm{~kg}$ de peso y con historia reciente de atropello vehicular. Se muestran las fracturas del cuerpo vertebral T12 y del proceso articular derecho (A), y severa inestabilidad de la articulación T12-T13 (B) (flechas) 


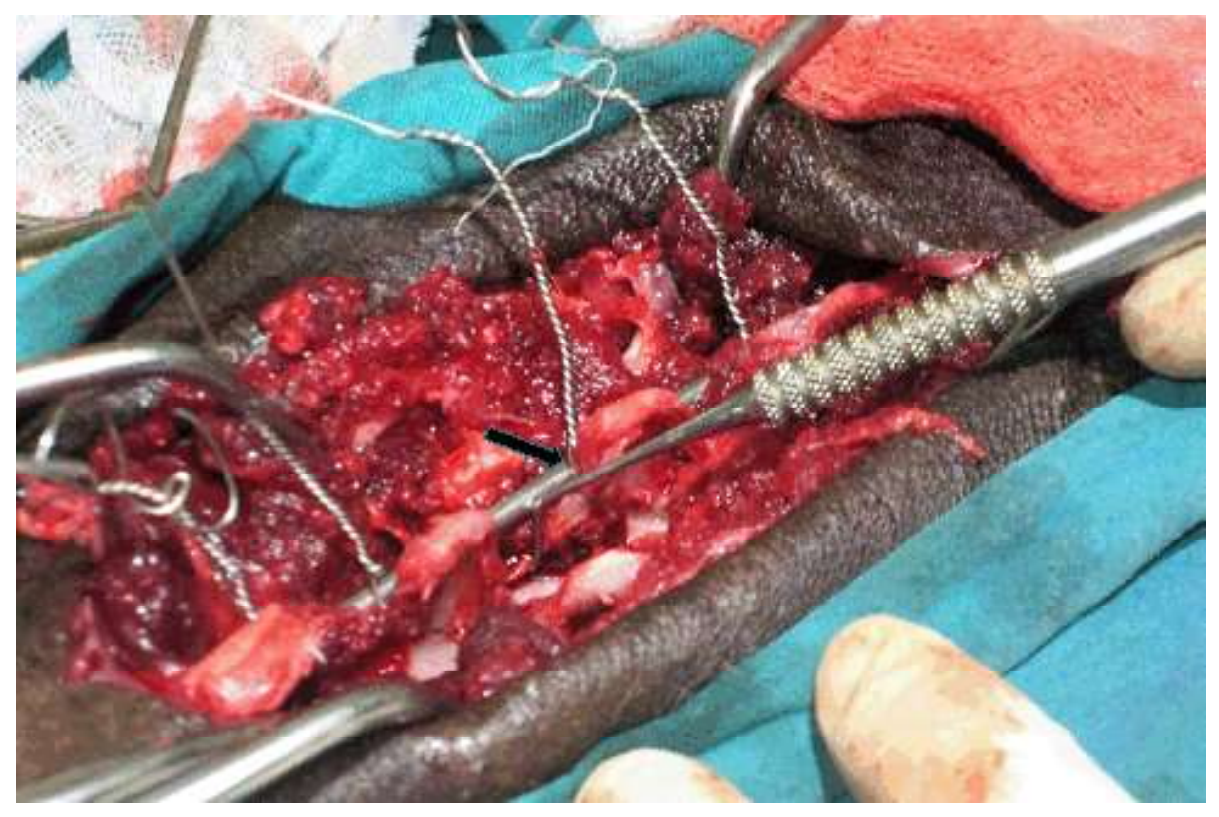

Figura 3. Exposición de los procesos espinosos de T11 a L1 durante el procedimiento quirúrgico en un perro de 2 años, con historia reciente de atropello vehicular. La flecha indica el clavo de Steinmann y el alambre quirúrgico utilizados en la fijación segmentaria

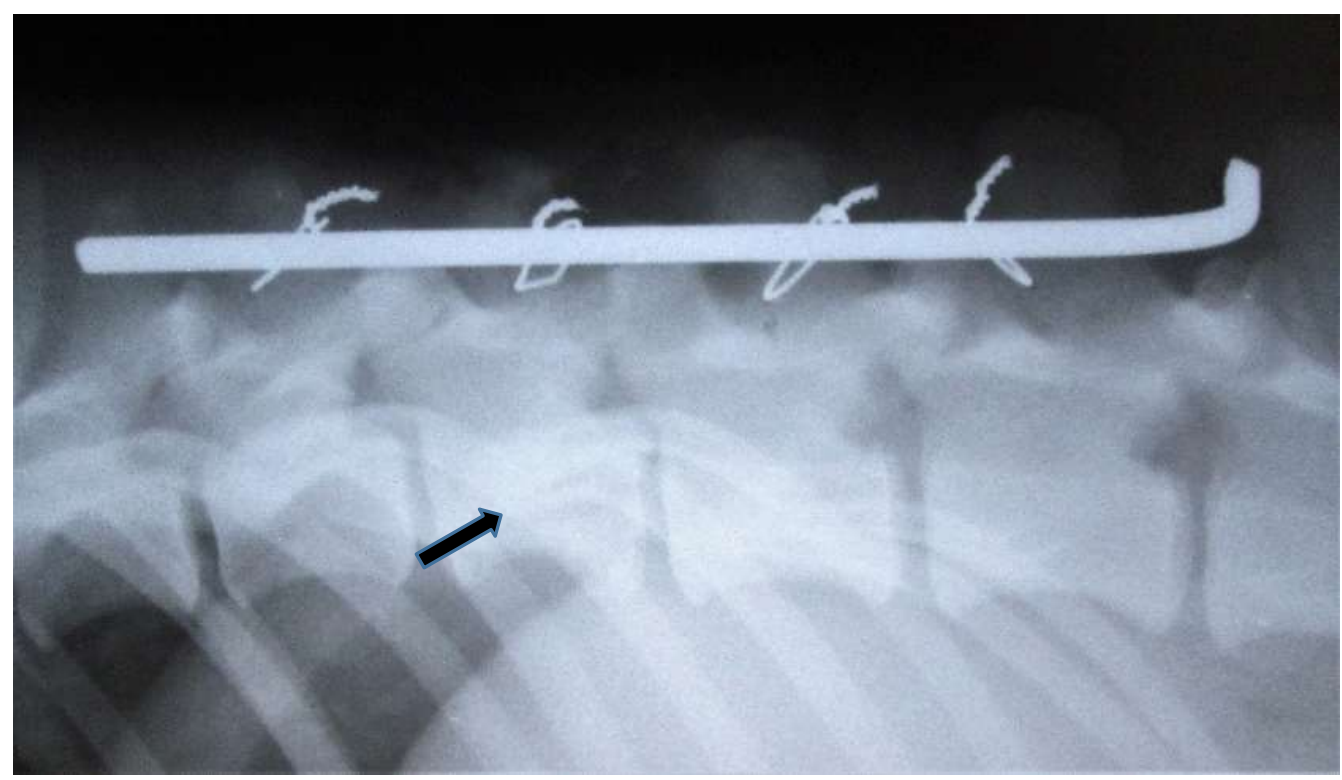

Figura 4. Imagen radiográfica laterolateral de la región intervenida quirúrgicamente de un perro de 2 años, con historia reciente de atropello vehicular. Se observa la estabilización de la luxofractura (flecha) y el realineamiento del eje vertebral 


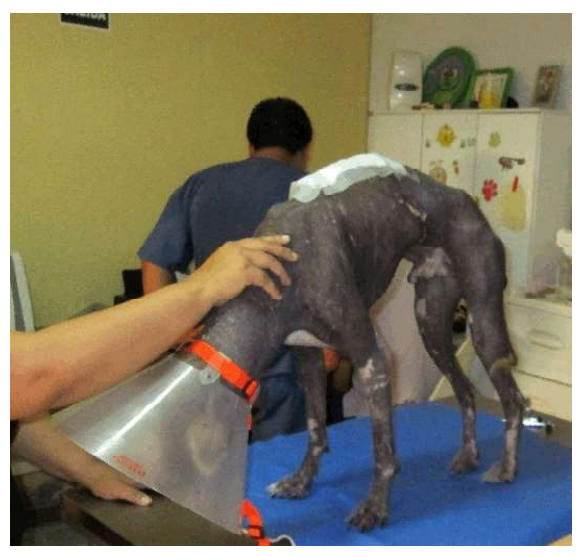

Figura 5. Imagen del paciente en la evaluación posquirúrgica a los siete días de la intervención. Se puede apreciar la recuperación de la postura.

comprobando mediante exploración interna la ausencia de estructuras compresivas del cordón medular dentro del canal, se llevó a cabo la estabilización vertebral mediante el uso de un clavo de Steinmann de $4 \mathrm{~mm}$ fijado por medio de alambres quirúrgicos de $0.8 \mathrm{~mm}$ a los procesos espinosos de las vértebras T11T12-T13 y L1, a través de agujeros pequeños practicados en la base de estos (Figura 3).

El extremo caudal del clavo de Steinmann fue curvado a fin de evitar su migración y malposición. Después de colocado el sistema de estabilización se realizó movimientos de compresión y distracción, además de rotación de la columna mostrándose un grado de estabilidad que se consideró aceptable. La fascia de los músculos epiaxiales se suturó con material absorbible (ácido poliglicólico 00) con un patrón de sutura continua de Ford. Las estructuras subcutáneas se afrontaron con un patrón de sutura continuo simple con el mismo material. Por último, la piel fue suturada con nylon 00 con un patrón de sutura continua de Ford. Al término de la intervención se obtuvo una imagen radiográfica en la que se observa el realineamiento de la columna vertebral y la correcta colocación de los implantes (Figura 4).

\section{Discusión}

Uno de los puntos cruciales en el manejo de pacientes caninos con trauma espinal es la obtención de información a partir de los signos clínicos (Bagley, 2003). En el presente caso, el diagnóstico clínico temprano permitió localizar la lesión a nivel toracolumbar y catalogarla como de grado 4 ante la escasa respuesta al estímulo doloroso y la incapacidad motora voluntaria (Sharp y Wheeler, 2005), además de mostrar el empeoramiento de la función medular al desaparecer la sensación de dolor profundo al día siguiente de la consulta.

La imagen radiográfica y tomográfica confirmaron el alto grado de inestabilidad de la fractura vertebral y condujeron a la conclusión de la necesidad de estabilización quirúrgica de la lesión (Jeffery, 2010; Vigano y Blasi, 2013). El peso moderado del paciente, el tiempo que había transcurrido desde el inicio de la lesión (menos de 24 horas) y los escasos medios económicos con los que contaba el propietario fueron puntos críticos respecto a la elección de la técnica quirúrgica a utilizarse.

En este caso se decidió la aplicación de la fijación segmentaria utilizando un clavo de Steinmann y alambres quirúrgicos, dado que constituye una técnica quirúrgica sencilla en la cual se emplean materiales de bajo costo que podrían cumplir con los objetivos de la estabilización de la luxofractura y, además, se puede usar en animales cuyo peso promedio se encuentra alrededor de los $10 \mathrm{~kg}$ (Voss y Montavon, 2004). McAnulty et al. (1986) utilizaron una variante de fijación segmentaria con clavo de Steinmann y alambre quirúrgico en cuatro pacientes caninos con luxofractura vertebral toracolumbar y lumbosacra entre 10 y $56 \mathrm{~kg}$, obteniendo estabilidad satisfactoria en todos los casos.

En pacientes humanos con trauma medular, se ha observado que cuando la cirugía se realiza dentro de las 24 horas desde el 
inicio del daño medular se obtiene mayor recuperación neurológica motora y acortamiento del tiempo de internamiento hospitalario (Verlaan et al., 2004; Dvorak et al., 2014).

Si bien algunos autores consideran que los clavos y alambres de acero quirúrgico presentan deficiencias en la estabilización biomecánica de columnas altamente inestables (Leone y Durante, 2012), en el presente caso se obtuvo la estabilidad necesaria para la corrección de la luxofractura. Por otro lado, el tamaño de los procesos espinosos fue adecuado para poder utilizar el método descrito y mantener estas estructuras sin que se ocasionen fracturas posquirúrgicas (Gage, 1971). En este paciente no se presentaron infecciones posquirúrgicas, lo cual en algunas ocasiones constituye un riesgo utilizando técnicas de fijación externa o con el empleo de cemento óseo como lo menciona Alegre et al. (1989) y Bruce et al. (2008).

Se puede deducir que la recuperación satisfactoria de la función medular del paciente estaría asociada a las acciones multidisciplinarias ejecutadas de manera oportuna, sin dejar de mencionar el poco tiempo transcurrido desde el momento del trauma y el peso moderado del paciente. La realización del examen neurológico y la obtención de imágenes permitieron el diagnóstico clínico temprano; el manejo y estabilización quirúrgica temprana de la lesión probablemente impidió el desarrollo del complejo proceso fisiopatológico del daño medular secundario (Butanda et al., 2013).

Es fundamental el conocimiento de la anatomía vertebral y la semiología medular, así como de los procesos fisiopatológicos del trauma medular agudo, para afrontar de manera exitosa la evaluación neurológica y el tratamiento de estos pacientes. Factores como la no evaluación constante de la respuesta del paciente después de la implementación de protocolos médicofarmacológicos o la aplicación de los mismos con bajo criterio diagnóstico llevan al escaso éxito de las medidas terapéuticas, y el desenlace es la pérdida de la función medular y, muchas veces, la eutanasia de animales que, probablemente, con un exhaustivo examen neurológico inicial, podrían llegar a ser tratados médica o quirúrgicamente en el momento y de la forma más adecuados.

\section{Literatura Citada}

1. Alegre F, Durán AJ, López A, Robert S. 1989. Fractura de quinta vértebra lumbar: nueva técnica de reducción. AVEPA 9: 161-164.

2. Bagley RS. 2000. Spinal fracture or luxation. Vet Clin NAm-Small 30: 133-153. doi: 10.1016/S0195-5616(00)50006-0

3. Bagley R. 2003. Trauma espinal. En: Pellegrino F, Suraniti A, Garibaldi L (eds). El libro de la neurología para la práctica clínica. Santa Fe, Argentina: Ed InterMédica. 720 p.

4. Bruce CW, Brisson BA, Gyselinck K. 2008. Spinal fracture and luxation in dogs and cats: a retrospective evaluation of 95 cases. Vet Comp Orthopaed 21: 280-284. doi: 10.1055/s-0037-1617373

5. Bulcão A, Da Paz Junior CJ, Silva RO. 2012. Estudo retrospectivo da ocorrência de fratura de coluna em cães e gatos atendidos no laboratório de radiologia da clínica veterinária da ESBAM- Manaus, AM. Amazon Sci 1: 25-27.

6. Butanda A, Guevara A, Matuz D, Rendón E, Vázquez H. 2013. Mecanismos de daño y neuroprotección en la lesión traumática de la médula espinal. Mensaje Bioquímico 37: 69-108.

7. Chipayo Y, Huerta C, Olazábal J, Díaz D. 2018. Tratamiento quirúrgico temprano de un caso de luxación patelar lateral bilateral en un paciente canino. Rev Inv Vet Perú 29: 720-724. doi: 10.15381/rivep.v29i2.14535 
8. Dvorak MF1, Noonan VK, Fallah N, Fisher CG, Finkelstein J, Kwon BK, Rivers CS, et al. 2014. The influence of time from injury to surgery on motor recovery and length of hospital stay in acute traumatic spinal cord injury: an observational Canadian cohort study. J Neurotraum 32: 645-654. doi: 10.1089/ neu. 2014.3632

9. Fossum TW, Hedlund CS, Johnson AL, Schulz KS, Seim HB, Willard MD, Carroll GL. 2007. Small animal surgery. $3^{\text {rd }}$ ed. EEUU: Mosby-Elsevier. $1610 \mathrm{p}$.

10. Gage ED. 1971. Surgical repair of spinal fractures in small breed dogs. Vet Med Small Anim Clin 66: 1095-1101.

11. Harrington P. 1962. Treatment of scoliosis. J Bone Joint Surg 44: 591-634.

12. Jeffery ND. 2010. Vertebral fracture and luxation in small animals. Vet Clin N Am-Small 40: 809-828. doi: 10.1016/ j.cvsm.2010.05.004

13. Kirby BM. 1995. Spinal fracture/luxation. Vet Clin NAm-Small 25: 1149-1174.

14. Leone FL, Durante EJ. 2012. Nuevo sistema de fijación especial en caninos. Analecta Vet 32: 24-32.

15. McAnulty JF, Lenehan TM, Maletz LM. 1986. Modified segmental spinal instrumentation in repair of spinal fractures and luxations in dogs. Vet Surg 15: 143-149. doi: 10.1111/j.1532-950X.1986.tb00192.x

16. Moshirfar A, Rand FF, Sponseller PD, Parazin SJ, Khanna AJ, Kebaish KM, Stinson JT, Riley LH III. 2005. Pelvic fixation in spine surgery. Historical- overview, indications, biomechanical relevance, and current techniques. $\mathrm{J}$ Bone Joint Surg Am. 87(Suppl 2): 89106. doi: 10.2106/JBJS.E. 00453

17. Pellegrino F. 2010. Fisiopatología del trauma medular agudo. En: Primeras jornadas de neurología veterinaria argentina. Buenos Aires: Asociación Argentina de Neurología Veterinaria.

18. Sharp N, Wheeler S. 2005. Thoracolumbar disc disease. In: Animal spinal disorders: diagnosis and surgery. $2^{\text {nd }} \mathrm{ed}$. Mosby. p 121-159.

19. Shores A. 1992. Spinal trauma: pathophysiology and management of traumatic spinal injuries. Vet Clin NAmSmall 22: 859-888. doi: 10.1016/S01955616(92)50080-8

20. Slatter D. 2006. Tratado de cirugía en pequeños animales. $3^{\mathrm{a}}$ ed. Buenos Aires, Argentina: Inter-Médica. 3156 p.

21. Verlaan JJ, Diekerhof CH, Buskens E, Tweel I, Verbout AJ, Dhert W, Oner FC. 2004. Surgical treatment of traumatic fractures of the thoracic and lumbar spine: a systematic review of the literature on techniques, complications, and outcome. Spine 29: 803-814. doi: 10.1097/01.BRS.0000116990.31984.A9

22. Vigano F, Blasi C. 2013. Valoración inicial de los traumatismos medulares. Vet Focus 23: 32-38.

23. Voss K, Montavon PM. 2004. Tension band stabilization of fractures and luxations of the thoracolumbar vertebrae in dogs and cats: 38 cases (1993-2002). J Am Vet Med Assoc 225: 78- 83. doi: 10.2460/javma.2004.225.78 